\title{
Construction of hypervesiculation Escherichia coli strains and application for secretory protein production
}

\section{Yoshihiro Ojima, Tomomi Sawabe, Katsuya Konami,}

\section{Masayuki Azuma}

\begin{tabular}{|c|l|}
\hline Citation & Biotechnology and Bioengineering. 117(3); 701-709. \\
\hline Issue Date & $2020-02-11$ \\
\hline Type & Journal Article \\
\hline Textversion & author \\
\hline Rights & $\begin{array}{l}\text { This is the pre-peer reviewed version of the following article: Biotechnology and } \\
\text { Bioengineering. Vol.117, Issu.3, p.701-709., which has been published in final form at } \\
\text { https://doi.org/10.1002/bit.27239. This article may be used for non-commercial } \\
\text { purposes in accordance with Wiley Terms and Conditions for Use of Self-Archived } \\
\text { Versions. }\end{array}$ \\
\hline DOI & $10.1002 /$ bit.27239 \\
\hline
\end{tabular}

\section{Self-Archiving by Author(s)}

Placed on: Osaka City University Repository 
Construction of hypervesiculation Escherichia coli strains and application for secretory protein production

Yoshihiro Ojima*, Tomomi Sawabe, Katsuya Konami and Masayuki Azuma*

Department of Applied Chemistry and Bioengineering, Graduate School of Engineering, Osaka City University, 3-3-138, Sugimoto, Sumiyoshi-ku, Osaka 558-8585, Japan

Keywords: Escherichia coli, outer membrane vesicle, gene deletion, protein secretion, OmpW, GFP

*Corresponding authors: Yoshihiro Ojima, Masayuki Azuma

Tel.: +81-6-6605-3092

Fax: +81-6-6605-3092

Email: ojima@osaka-cu.ac.jp, azuma@osaka-cu.ac.jp 


\section{Abstract}

Outer membrane vesicles (OMVs) are extracellular vesicles released from the

3 surface of Gram-negative bacteria, including Escherichia coli. Several gene-deficient

4 mutants relating to envelope stress ( $n l p I$ and $\operatorname{deg} P$ ) and phospholipid accumulation in

5 the outer leaflet of the outer membrane (mlaA and mlaE) increase OMV production.

6 This study examined the combinatorial deletion of these genes in E. coli and its effect

7 on OMV production. The $n l p I$ and mlaE double-gene-knockout mutant ( $\Delta$ mlaE $\Delta n l p I)$

8 showed the highest OMV production. Sodium dodecyl sulfate-

9 polyacrylamide gel electrophoresis-based quantitative analysis showed that OMV

10 production by strain $\Delta$ mlaE $\Delta$ nlpI was $\sim 30$ times that by the wild-type. In addition, to

11 evaluate the protein secretion capacity of OMVs, green fluorescent protein (GFP) fused

12 with outer membrane protein $\mathrm{W}(\mathrm{OmpW})$ was expressed in OMVs. Western blot

13 analysis showed that GFP secretion through OMVs reached $3.3 \mathrm{mg} / \mathrm{L}$ in the culture

14 medium of strain $\Delta m l a E \Delta n l p I / g f p, 500$ times that for the wild-type. Our approach using

15 OMVs for extracellular protein secretion in E. coli is an entirely new concept compared 16 with existing secretion systems. 


\section{Introduction}

Outer membrane vesicles (OMVs) are nanosized (20-250 nm diameter), spherical bilayered proteolipids that are normally discharged from the surface of Gram-negative bacteria, including Escherichia coli (Schwechheimer and Kuehn, 2015; Toyofuku et al., 2019). OMVs contain outer membrane proteins, lipids, periplasmic proteins, lipopolysaccharides, RNA, and DNA. Studies have reported that disturbances in growth, turnover in cell wall components, or exposure to antibiotics initiate vesicle formation (Schwechheimer and Kuehn, 2015; Toyofuku et al., 2019). In particular, envelope stress and phospholipid accumulation in the outer membrane are gaining attention as dominant endogenous factors triggering OMV production by Gram-negative bacteria.

In $E$. coli, deletion of the genes $\operatorname{deg} P$ or $n l p I$ induces envelope stress and OMV production (Schwechheimer et al., 2015). The $\operatorname{deg} P$ gene encodes a periplasmic protease/chaperone that manages unfolded and misfolded periplasmic proteins (Lipinska et al., 1990). Enhanced OMV production in the $\Delta \operatorname{deg} P$ strain is a possible survival strategy that eliminates these undesired proteins from the cell envelope (Schwechheimer and Kuehn, 2013). Recent studies have correlated vesiculation levels with covalent envelope cross-linking. The amount of lipoprotein-peptidoglycan (LppPG) crosslinks is $\sim 40 \%$ lower in the hypervesiculating $n l p I$ mutant compared with the wild-type (WT) strain (Schwechheimer et al., 2015). It is believed that the breakdownsynthesis balance of PG is modified in the nlpI mutant, preventing the formation of proper crosslinks and indirectly leading to OMV production (Schwechheimer and Kuehn, 2015). OMV production is also closely related to the phospholipid transport system. Roier et al. (2016) showed that dysfunction of VacJ (also known as MlaA) and the Yrb ATP-binding cassette $(\mathrm{ABC})$ transport system increases OMV production in the 
1 Gram-negative bacteria Haemophilus influenzae and Vibrio cholerae because of

2 phospholipid accumulation in the outer leaflet of the outer membrane (Roier et al.,

3 2016). This observation was confirmed by a genome-wide assessment of OMV

4 production in E. coli, indicating increased vesiculation of mlaA (vacJ) and mlaE (yrbE)

5 mutants (Kulp et al., 2015). Therefore, both envelope stress and phospholipid

6 accumulation are important endogenous factors promoting OMV production. However,

7 it is unclear how combinatorial deletion of such genes affects OMV production in $E$.

8 coli.

9 Bacterial OMVs have received considerable attention because of their applicability

10 in biotechnology. The bacterial cellular machinery can be genetically engineered to

11 produce and package heterologous enzymes in OMV nanoparticles. (Alves et al., 2015;

12 Alves et al., 2016). In our previous study, outer membrane protein W (OmpW)

13 efficiently packaged green fluorescent protein (GFP) into OMVs of E. coli (WT strain

14 and hypervesiculating mutants $\Delta d e g P$ and $\Delta n l p I$ ) by overexpression of an OmpW-GFP

15 fused protein (Ojima et al., 2018). The $\Delta n l p I$ strain showed higher OMV production and

16 GFP secretion into the culture medium than the $\Delta \operatorname{deg} P$ strain (Ojima et al., 2018).

17 Therefore, using OMVs as a protein secretion tool in microbial engineering is

18 interesting.

19 In this study, we examined combinatorial deletion of genes involved in envelope

20 stress ( $\operatorname{deg} P$ and $n l p I)$ and phospholipid accumulation (mlaA and mlaE) in E. coli and its

21 effect on OMV production. To evaluate the protein secretion capacity of OMVs, GFP

22 was packaged into the OMVs. We investigated the application potential of OMVs as a

23 protein secretion tool in E. coli. 
1

2

3

4

5

6

7

\section{Materials and Methods}

\subsection{Bacterial strains and culture conditions}

Table 1 lists the strains and plasmids used in this study. The E. coli K-12 strain BW25113 (WT) and its derivatives were obtained from the National BioResource Project (National Institute of Genetics [NIG], Mishima, Japan) (Baba et al., 2006).

Double-gene knockout mutants were constructed by P1 transduction using P1kc phage (Koma et al., 2012). Briefly, the kanamycin $(\mathrm{Km})$ cassette was eliminated from the recipient single-gene knockout mutant using flippase/flippase recognition target (Flp/FRT) recombination. Then, P1 transduction was performed on a Km-sensitive recipient strain, with the donor strain harboring an FRT-Km-FRT cassette, which was inserted at the desired locus on the chromosome. The recipient strain was selected by Km resistance. ASKA-omp $W$-gfp was obtained from NIG (Kitagawa et al., 2005), and transformants containing ASKA-omp $W$ - $g f p$ were named in the style WT/gfp.

E. coli cells were cultured in lysogeny broth (LB; $10 \mathrm{~g} / \mathrm{L}$ Bacto ${ }^{\mathrm{TM}}$ Tryptone, $5 \mathrm{~g} / \mathrm{L}$ yeast extract, and $10 \mathrm{~g} / \mathrm{L} \mathrm{NaCl}$ ). The culture medium of strains harboring ASKA-omp $W$-gfp was supplemented with $50 \mathrm{mg} / \mathrm{L}$ chloramphenicol. All test cultures were precultured in $\mathrm{LB}$ for $18 \mathrm{~h}$ at $37^{\circ} \mathrm{C}$ and inoculated into $100 \mathrm{~mL}$ fresh $\mathrm{LB}$ in a $500-\mathrm{mL}$ baffled conical flask such that the optical density at $660 \mathrm{~nm}\left(\mathrm{OD}_{660}\right)=0.01$. The cultures were placed on an NR-30 rotary shaker (Taitec, Osaka, Japan), with shaking at 140 strokes/min. Cell growth was recorded by measuring OD 660 values.

\subsection{Gene expression analysis}

To examine gene expression, cells of each strain were harvested at 4 and $8 \mathrm{~h}$ post-inoculation by centrifugation at $4^{\circ} \mathrm{C}$ for $10 \mathrm{~min}$ at $8,000 \times g$. Total RNA was 
extracted from the collected cells as described elsewhere (Ojima et al., 2018), and then reverse-transcribed into cDNA using a PrimeScript RT reagent kit (Takara Bio Inc., Kusatsu, Japan). Gene expression was analyzed by real-time PCR (Mx3000P, Agilent Technology, Santa Clara, CA), as described in our previous study (Ojima et al., 2018). The gene expression level of omp $W-g f p$ was normalized against that of $r r s A$ (16S rRNA). The specific primer pairs used are listed in Table 1.

\subsection{Isolation and transmission electron microscope (TEM) observation of OMVs}

OMVs were isolated, as previously described (Gujrati et al., 2014) with some modifications. Briefly, after incubation for $24 \mathrm{~h}, 100 \mathrm{~mL}$ of $E$. coli culture broth were centrifuged at $10,000 \times g$ for $10 \mathrm{~min}$ at $4^{\circ} \mathrm{C}$ to remove the cells. Then, the supernatant was passed through a $0.45-\mu \mathrm{m}$ pore filter. Ammonium sulfate was added (final concentration, $400 \mathrm{~g} / \mathrm{L}$ ) for $1 \mathrm{~h}$ at room temperature to precipitate the contents. Crude OMVs were obtained by centrifugation at $11,000 \times g$ for $30 \mathrm{~min}$ at $4^{\circ} \mathrm{C}$. The crude extracts were dissolved in $500 \mu \mathrm{L}$ of $15 \%(\mathrm{v} / \mathrm{v})$ glycerol solution and then concentrated using a CS100FNX ultracentrifuge (Hitachi Koki Co., Tokyo, Japan) at 109,000 $\times g$ for $1 \mathrm{~h}$. The OMV pellets were resuspended in $100 \mu \mathrm{L}$ of $15 \%$ (v/v) glycerol solution. The resulting OMV samples were 1000 times more concentrated compared with the original culture broth because of a decrease in the volume from $100 \mathrm{~mL}$ to $100 \mu \mathrm{L}$. The OMV samples were dropped onto a mesh copper grid and negatively stained with $4 \%$ uranyl acetate for observation under a JEM-2100 TEM (JEOL, Tokyo, Japan).

\subsection{Evaluation of OMV production and GFP secretion through OMVs}

We took $10-\mu \mathrm{L}$ samples of the isolated OMVs or E. coli cells of each strain for 
1 analysis using sodium dodecyl sulfate-polyacrylamide gel electrophoresis (SDS-PAGE)

2 with Coomassie Blue staining. OMV production was quantified as previously described

3 (Schwechheimer and Kuehn, 2013) with some modifications. To index the OMV

4 concentration, we photographed the SDS-PAGE band at $\sim 37 \mathrm{kDa}$ and analyzed the

5 densitometry using Image J software (National Institutes of Health, Bethesda, MD,

6 USA). The index was normalized against OMV production by the WT strain. OMVs

7 were also quantified on the basis of phospholipid, with minor modifications from a

8 previously described method (Klimentová and Stulík, 2015; Manning and Kuehn, 2011;

9 McBroom et al., 2006). Briefly, isolated OMVs were incubated with FM4-64

10 (Molecular Probes/Thermo Fisher, IL, USA) at a final concentration of $5 \mu \mathrm{g} / \mathrm{mL}$ in 11 phosphate-buffered saline (pH 7.4) for $20 \mathrm{~min}$. We used samples without OMVs or without FM4-64 as negative controls. After excitation at $558 \mathrm{~nm}$, emission at $734 \mathrm{~nm}$ was measured using an INFINITE 200 PRO spectrofluorophotometer (TECAN, Switzerland).

For western blot analysis, we transferred protein from a gel to an Immobilon-P membrane sheet (Merck Millipore, Billerica, MA, USA) using the semidry transfer method. Hybridization was performed using an anti-histidine-tag monoclonal antibody as the primary antibody (Medical \& Biological Laboratories Co., Nagoya, Japan) and anti-mouse immunoglobulin G (whole molecule)-alkaline phosphatase (Sigma-Aldrich, St. Louis, MO, USA) as the secondary antibody. Hybridization signals were detected using a BCIP-NBT Solution Kit for Alkaline Phosphatase Stain (Nacalai Tesque, Kyoto, Japan). To index target protein expression, we photographed the western blot band and analyzed it using densitometry. GFP secretion was quantified on the basis of a GFP standard sample with a His-tag. GFP secretion was also quantified by fluorescence 
1 measurement. The fluorescence intensity of GFP in OMVs was measured by emission at $2510 \mathrm{~nm}$ after excitation at $450 \mathrm{~nm}$ using an INFINITE 200 PRO 3 spectrofluorophotometer.

$5 \quad 3$ Results and Discussion

\section{$6 \quad 3.1$ OMV production in single- and double-gene knockout mutants}

Figure 1A shows the cell growth of each single- and double-knockout mutant of $E$. coli at the end of culture for $24 \mathrm{~h}$. $\mathrm{OD}_{660}$ of the WT strain was 5.1. Among 9 single-knockout mutants, $\mathrm{OD}_{660}$ of the $\Delta n l p I$ and $\Delta d e g P$ strains decreased slightly compared with that of the WT, as reported previously (Ojima et al., 2018). However, mlaE deletion did not affect cell growth. Overall, these results suggest that deleting single genes involved in envelope stress and phospholipid accumulation does not drastically influence E. coli cell growth.

14 In contrast, several double-gene and triple-gene knockout mutants showed significantly lower cell growth than the WT. $\mathrm{OD}_{660}$ of the $\Delta \operatorname{degP} \Delta m l a E, \Delta$ nlpI $\Delta$ mlaA, $\Delta d e g P \Delta n l p I$ and $\Delta m l a E \Delta n l p I \Delta d e g P$ strains was $\sim 3.5$; the $\Delta m l a E \Delta n l p I$ strain had the lowest $\mathrm{OD}_{660}$ (2.9). Therefore, combinatorial gene knockout represses E. coli cell growth.

To analyze OMV production, OMVs in supernatants were obtained from the culture broth of respective strains after $24 \mathrm{~h}$ by ultracentrifugation. In E. coli samples, SDS-PAGE bands observed at $\sim 37 \mathrm{kDa}(\mathrm{OmpF}$, OmpC, and OmpA) provide an index of OMV production, as these membrane proteins are expressed specifically and abundantly within the outer membrane of cells and, therefore, predominate in OMVs

24 (Schwechheimer and Kuehn, 2013). Figure 1B shows the SDS-PAGE band of each 
1 OMV sample. Results clearly confirmed the presence of SDS-PAGE bands at $\sim 37 \mathrm{kDa}$

2 in samples from all strains, indicating that OMVs were successfully isolated from the

3 culture broth. OmpA, OmpC, and OmpF were identified from these bands by MALDI

4 FT-ICR MS in preliminary experiments using the WT strain (data not shown). Next, we

5 quantitatively analyzed OMV production on the basis of densitometry of the $\sim 37 \mathrm{kDa}$

6 SDS-PAGE bands (Fig. 1C). $\Delta n l p I$ and $\Delta d e g P$ strains produced, respectively, $\sim 7$ and 5

7 times more OMVs than the WT. These results are consistent with those of previous

8 studies (Ojima et al., 2018; Schwechheimer et al., 2015). The OMV production by

$9 \Delta m l a E$ and $\triangle m l a A$ strains was, respectively, $\sim 5$ and 3 times higher compared with the

WT. The increase in vesiculation upon gene deletion in the Mla pathway is consistent with previous reports (Kulp et al., 2015; Roier et al., 2016).

Among double-gene-knockout mutants, only the $\Delta m l a E \Delta m l a A$ strain did not show a further increase in OMV production compared with the single-gene-knockout mutants (Fig. 1C). This is the first study showing that a cumulative gene deletion in the Mla pathway is not effective in increasing OMV production. However, in contrast, other double-gene deletions did significantly increase OMV production compared with single-gene deletions, to $>15$-fold the level for the WT strain. The $\Delta$ mlaE $\Delta n l p I$ strain showed the highest OMV production, 30 times that by the WT. Studies have reported that increased OMV production following $n l p I$ deletion is due to prevention of the formation of proper Lpp-PG crosslinks (Schwechheimer et al., 2015). In contrast, dysfunction of the VacJ/Yrb ABC transport system increases OMV production because of phospholipid accumulation in the outer leaflet of the outer membrane (Roier et al., 2016). Our results demonstrated that combining PG breakdown and phospholipid accumulation in the outer leaflet of the outer membrane is effective in increasing OMV 
1 production by $E$. coli. In addition, given that the $\Delta m l a E \Delta n l p I$ strain had the lowest OD

2 at the end of culture, a strong increase in OMV production represses cell activity in $E$.

3 coli. A triple-gene-knockout mutant $(\Delta m l a E \Delta n l p I \Delta d e g P)$ was also examined. The $\mathrm{OD}_{660}$

4 of strain $\Delta m l a E \Delta n l p I \Delta \operatorname{deg} P$ was 3.4 and the OMV production was 13.9 -fold that by the

5 WT, suggesting that further cumulative gene deletion did not enhance the OMV

6 production. Figure 2 shows TEM images of isolated OMVs from the WT and the

7 double-gene-deletion mutant with the highest OMV production, i.e. $\Delta m l a E \Delta n l p I$. OMV

8 particles $\sim 20-100 \mathrm{~nm}$ in diameter were observed for both strains, suggesting that the

$9 \Delta m l a E \Delta n l p I$ strain produced OMVs in the culture broth.

\subsection{OMV production with GFP expression in double-gene-knockout mutants}

Previously, we demonstrated that OmpW-GFP fused protein shows excellent efficiency in packaging GFP into OMVs from E. coli (Ojima et al., 2018). In addition, the $\Delta n l p I / g f p$ strain showed higher efficiency in GFP secretion through OMVs than the $\Delta \operatorname{deg} P / g f p$ strain. In the present study, we introduced OmpW-GFP fused protein into double-gene knockout mutants. Figure 3A shows $\mathrm{OD}_{660}$ values of WT and double-gene knockout mutants carrying the ASKA-ompW-gfp plasmid. $\mathrm{OD}_{660}$ of the $\Delta \operatorname{degP} \Delta m l a A / g f p$ strain was comparable to that of the WT/gfp and $\Delta \operatorname{degP} \Delta m l a E / g f p$ strains, whereas the $\Delta m l a E \Delta m l a A / g f p$ and $\Delta n l p I \Delta m l a A / g f p$ strains grew to a slightly lower $\mathrm{OD}_{660}$ than the $\mathrm{WT} / g f p$ strain. Cell growth of the $\Delta d e g P \Delta n l p I / g f p$ and $\Delta m l a E \Delta n l p I / g f p$ strains was significantly lower than that of the WT/gfp strain, and the $\Delta m l a E \Delta n l p I / g f p$ strain showed the lowest $\mathrm{OD}_{660}$ (3.6). These results, in comparison with the data in Fig. 1A, suggested that introducing the ASKA-omp $W$-gfp plasmid did 
1 not greatly change the cell growth tendency of the WT or double-gene-knockout

2 mutants, although there was a slight overall increase.

We quantitatively estimated OMV production in recombinant double-gene knockout mutants on the basis of densitometry of the $\sim 37-\mathrm{kDa}$ SDS-PAGE bands (Fig. 3B). Whereas the $\triangle \operatorname{deg} P \Delta m l a E$ and $\triangle \operatorname{deg} P \Delta$ mlaA strains showed $\sim 20$ times higher OMV production than the WT (Fig. 1C), $\Delta \operatorname{deg} P \Delta m l a E / g f p$ and $\Delta \operatorname{deg} P \Delta m l a A / g f p$ strains showed, respectively, $\sim 5$ and 3 times more OMV production than the WT/gfp strain (Fig. 3B). These results suggested that introducing the ASKA-omp $W$-gfp plasmid represses OMV production in these double-gene-knockout mutants. In contrast, strains $\Delta n l p I \Delta m l a A / g f p, \Delta \operatorname{degP} \Delta n l p I / g f p$, and $\Delta m l a E \Delta n l p I / g f p$ produced more OMVs than other strains; notably, the nlpI gene was deleted from all three of these mutants. In particular, OMV production by strain $\Delta$ mlaE $\Delta n l p I / g f p$ was found to be $\sim 40$ times that by the WT. The combinatorial deletion of $m l a E$ and $n l p I$ is effective in increasing OMV production by $E$. coli regardless of plasmid introduction.

\subsection{GFP secretion through OMVs}

As shown in Fig. 4A, western blot bands appeared at the expected molecular mass of OmpW-GFP fused protein $(\sim 50 \mathrm{kDa})$ in all lanes, suggesting that it was properly expressed in OMVs. In a preliminary experiment using the WT/gfp strain, OmpW-GFP was identified from the corresponding SDS-PAGE band by MALDI FT-ICR MS (data not shown). In single-gene-knockout mutants, the western blot band was much more intense for the $\Delta n l p I / g f p$ strain than for the WT/gfp strain, even though the $\Delta n l p I / g f p$ strain sample was diluted 50 times. In double-gene-knockout mutants, with the dilution of each sample taken into consideration, $\Delta n l p I \Delta m l a A / g f p, \Delta d e g P \Delta n l p I / g f p$, and 
$1 \Delta m l a E \Delta n l p I / g f p$ strains secreted much more GFP compared with other strains. The

2 amount of GFP secreted through OMVs in the culture medium was quantified on the

3 basis of densitometry of the western blot band from commercial GFP with known

4 concentration. As shown in Fig. 4B, the WT/gfp strain secreted $6.6 \mu \mathrm{g} / \mathrm{L}$ of GFP through

$5 \quad$ OMVs into the culture medium. GFP secretion by strains $\Delta n l p I$ and $\Delta \operatorname{deg} P$ was 95.1

6 and $35.6 \mu \mathrm{g} / \mathrm{L}$, respectively, significantly higher than by the WT. Considering

7 double-gene-knockout mutants, GFP secretion by strains $\Delta \operatorname{deg} P \Delta m l a E / g f p$,

$8 \Delta \operatorname{deg} P \Delta m l a A / g f p$, and $\Delta$ mlaE $\Delta m l a A / g f p$ was less than that by the single-gene knockout

9 mutant $\Delta n l p I / g f p$ because the OMV production by these strains was strongly repressed

10 by the introduction of the ASKA-omp $W$-gfp plasmid (Fig. 3B). In contrast, three

11 double-gene-knockout mutants from which nlpI was deleted secreted a significantly

12 higher amount of GFP; strains $\Delta n l p I \Delta m l a A / g f p, \Delta d e g P \Delta n l p I / g f p$, and $\Delta m l a E \Delta n l p I / g f p$

13 respectively secreted $1.7,2.5$ and $3.3 \mathrm{mg} / \mathrm{L}$ GFP, up to 500 times higher than that by the

14 WT.

15 Considering that the relative OMV production by strain $\Delta$ mlaE $\Delta n l p I / g f p$ was 40 times that by the WT/gfp strain as determined by SDS-PAGE, a 500 -fold increase in

17 GFP secretion cannot be completely explained by increased OMV production (Table 2).

18 In addition to evaluation of GFP secretion using western blot analysis,

19 fluorescence-based measurement again showed $3.3 \mathrm{mg} / \mathrm{L}$ of GFP secretion in the culture

20 of strain $\Delta m l a E \Delta n l p I / g f p$. Therefore, we hypothesized that besides enhancing the OMV

21 production, deleting both mlaE and $n l p I$ influenced the level of GFP production in the $E$. coli cell. Supplementary Figure S1 shows western blot analysis, where the same amounts of whole-cell pellets were loaded to compare the GFP production in the cells.

As reported previously (Ojima et al., 2018), the band was more intense in $\Delta n l p I / g f p$ 
1 cells than in WT cells. Also, the band in $\Delta m l a E \Delta n l p I / g f p$ cells was about twofold the

2 intensity of that in WT cells, suggesting that GFP production in $\Delta m l a E \Delta n l p I / g f p$ cells is

3 approximately double that in the WT. Thus, the GFP secretion through OMV by strain

$4 \Delta$ mlaE $\Delta n l p I / g f p$ was increased not only by increased OMV production but also by

5 increased GFP expression per unit cell. However, a 500-fold increase in GFP secretion

6 was not completely explained. We also used real-time PCR analysis to determine the

7 mRNA expression of the ompW-gfp gene in WT and $\Delta m l a E \Delta n l p I$. Relative mRNA

8 expression of ompW-gfp in $\Delta m l a E \Delta n l p I$ compared with WT was $1.4 \pm 0.6$ at $4 \mathrm{~h}$ and

$9 \quad 1.3 \pm 0.8$ at $8 \mathrm{~h}$. Therefore, the transcription level was not significantly different

10 between the WT and $\Delta$ mlaEAnlpI mutant, suggesting that the increased amount of

11 OmpW-GFP in the OMV fraction of the $\Delta$ mlaEsnlpI mutant was not due to a dramatically increased transcription level of the $o m p W-g f p$ gene. protein and phospholipid balance of the OMVs and that SDS-PAGE analysis thus underestimates OMV production. So, OMV production was also compared among strains WT/gfp, $\Delta m l a E / g f p, \Delta n l p I / g f p$, and $\Delta m l a E \Delta n l p I / g f p$ on the basis of the amount of phospholipid. As shown in Table 2, relative OMV production by strain $\Delta n l p I / g f p$ determined using FM4-64 was 10.9 times that by the WT/gfp strain, corresponding to the result obtained by SDS-PAGE. However, strain $\Delta m l a E / g f p$ showed 9.6 times higher OMV production, whereas the value determined by SDS-PAGE was 1.7. With regard to this difference, it was reported that Mla pathway mutants increased OMV production because of phospholipid accumulation in the outer leaflet of the outer membrane (Roier et al., 2016). Therefore, we suggest that protein-based evaluation underestimated OMV production by strain $\Delta m l a E / g f p$. Meanwhile, phospholipid-based evaluation determined 
$1 \sim 15$ times higher OMV production by strain $\Delta m l a E \Delta n l p I / g f p$ compared with WT/gfp,

2 whereas the value determined by SDS-PAGE was $\sim 40$ times. Considering these values,

3 it can be suggested that OMV production by strain $\Delta m l a E \Delta n l p I / g f p$ is at least $\sim 15$ times

4 higher than that by the WT. Therefore, evaluation by SDS-PAGE did not underestimate

5 OMV production by strain $\Delta m l a E \Delta n l p I / g f p$, and underestimation of OMV production

6 by SDS-PAGE was not the reason for the nonlinearly of GFP secretion against OMV

7 production.

To check for the presence of free GFP in OMV fraction from strain $\Delta m l a E \Delta n l p I / g f p$,

9 OMV samples were treated by spin-filtration using a desalting column, 100K MWCO

10 (Amicon Ultra, Merck Millipore). The resultant samples were analyzed by western blot using an anti-GFP antibody. GFP was not detected in the flow through of the spin-filtered sample, suggesting that free GFP is not present in the OMV sample. Further studies are required to explain the additional increase in GFP secretion through OMVs in strain $\Delta m l a E \Delta n l p I / g f p$.

Quantitative estimation of GFP production in the cells is summarized in Table 2. GFP production in $\Delta m l a E \Delta n l p I / g f p$ cells was $261 \mathrm{mg} / \mathrm{L}$, which was almost equal to that in WT cells $(269 \mathrm{mg} / \mathrm{L})$ because $\mathrm{OD}_{660}$ of the $\Delta m l a E \Delta n l p I / g f p$ strain was around half that of the WT. The percentage of secreted GFP was calculated as $0.003 \%$ in WT/gfp strain culture and approximately $1.3 \%$ in $\Delta m l a E \Delta n l p I / g f p$ culture, suggesting a significant increase of GFP secretion in the double-gene-knockout mutant.

\subsection{Possibility of OMVs as a tool for protein secretion}

A recent study summarized the development of Gram-negative bacteria, including $E$. coli, for the secretion of heterologous proteins (Burdette et al., 2018). Although E. coli 
1 is a robust, inexpensive expression host for recombinant protein production, the typical

2 production process involves intracellular protein expression, which requires cell lysis

3 and multiple purification steps. Secretion of heterologous proteins into the extracellular

4 medium increases product purity from cell culture, decreases downstream processing

5 requirements, and reduces overall cost; therefore, the utility of E. coli would expand if it

6 secreted the protein product. Protein secretion from E. coli operates through highly

7 specialized systems that can translocate proteins from the cytosol to the extracellular

8 medium in either one or two steps (Burdette et al., 2018). The secretion systems for

9 native proteins in E. coli are categorized into types I, II, V, and VIII, and these systems

10 also successfully secrete recombinant proteins (Burdette et al., 2018).

11 Table 3 shows a list of heterologous proteins secreted by E. coli. For proteins with a relatively large molecular mass $(>30 \mathrm{kDa}), 2-30 \mathrm{mg} / \mathrm{L}$ are secreted, mainly by the type I secretion system (Fernandez et al., 2000; Khosa et al., 2018). The amount of GFP secreted by OMVs in this study $(3.3 \mathrm{mg} / \mathrm{L})$ is comparable with these values. The type I secretion system involves one-step secretion achieved by a HlyA-tagged guide. In this pathway, the protein cargo must be either completely or partially unfolded to be secreted. In the case of secretion of peptides or smaller proteins, studies have reported extremely high production (Browning et al., 2017; Chen et al., 2014), for example $6 \mathrm{~g} / \mathrm{L}$ of 13.1-kDa Fab (Voulgaris et al., 2015). Small proteins or peptides are secreted into the periplasm by the type II secretion system with extracellular secretion using a leaky outer membrane. The type V and VIII systems were applied for secretion of pertactin (1 $\mathrm{mg} / \mathrm{L}$ ) and cecropin A (294 mg/L after purification) (Sevastsyanovich et al., 2012; Wang et al., 2017), respectively. Compared with the existing secretion systems, our approach for extracellular protein secretion from E. coli using OMVs is an entirely new concept. 


\section{Conclusions}

E. coli strain $\Delta$ mlaEAnlpI showed the highest OMV production, $\sim 30$ times higher

4 than that by the WT strain. GFP secretion through OMVs reached $\sim 3 \mathrm{mg} / \mathrm{L}$ in the

5 culture medium of strain $\Delta m l a E \Delta n l p I$. Extracellular protein secretion using OMVs is an

6 entirely new concept compared with the existing secretion systems in E. coli.

7 Considering the downstream purification of recombinant protein from OMVs, it is

8 already confirmed that ultrasonication is effective for disruption of OMVs. Therefore,

9 insertion of protease site-specific cleavage sequences between $\mathrm{OmpW}$ and the target

10 protein will enable a smart purification process for the target protein.

\section{Acknowledgment}

13 The authors acknowledge financial support by a Grant-in-Aid for Young Scientists 14 (B) (No. 17K14869) from the Ministry of Education, Culture, Sports, Science and 15 Technology of Japan and a Chemical Innovation Encouragement Prize from the Japan 16 Association for Chemical Innovation. The authors also thank Dr. Koma and Dr. Ohmoto,

17 Osaka Institute of Industrial Science and Technology, for technical support in P1 18 transduction. We thank James Allen, DPhil, from Edanz Group 19 (www.edanzediting.com/ac) for editing a draft of this manuscript.

\section{Conflicts of interest}

22 The authors declare that they have no conflicts of interest. 
YO and MA proposed the research concept and provided necessary tools for experiments and experimental instructions. YO designed experiments, analyzed data and wrote the manuscript. TS and KK conducted experiments and analyzed data. All authors read and approved the manuscript.

\section{Literature Cited}

Alves, N. J., Turner, K. B., Daniele, M. A., Oh, E., Medintz, I. L., Walper, S. A. (2015). Bacterial nanobioreactors - directing enzyme packaging into bacterial outer membrane vesicles. ACS Applied Materials \& Interfaces, 7(44), 24963-24972.

Alves, N. J., Turner, K. B., Medintz, I. L., Walper, S. A. (2016). Protecting enzymatic function through directed packaging into bacterial outer membrane vesicles. Scientific Reports, 6, 24866.

Baba, T., Ara, T., Hasegawa, M., Takai, Y., Okumura, Y., Baba, M., Datsenko, K. A., Tomita, M., Wanner, B. L., Mori, H. (2006). Construction of Escherichia coli K-12 in-frame, single-gene knockout mutants: The Keio collection. Molecular Systems Biology, 2, 2006.0008.

Browning, D. F., Richards, K. L., Peswani, A. R., Roobol, J., Busby, S. J. W., Robinson, C. (2017). Escherichia coli "TatExpress" strains super-secrete human growth hormone into the bacterial periplasm by the Tat pathway. Biotechnology and Bioengineering, 114(12), 2828-2836.

Burdette, L. A., Leach, S. A., Wong, H. T., Tullman-Ercek, D. (2018). Developing Gram-negative bacteria for the secretion of heterologous proteins. Microbial Cell Factories, 17(1), 196.

Chen, Z. Y., Cao, J., Xie, L., Li, X. F., Yu, Z. H., Tong, W. Y. (2014). Construction of 
leaky strains and extracellular production of exogenous proteins in recombinant Escherichia coli. Microbial Biotechnology, 7(4), 360-370.

Fernandez, L. A., Sola, I., Enjuanes, L., de Lorenzo, V. (2000). Specific secretion of active single-chain Fv antibodies into the supernatants of Escherichia coli cultures by use of the hemolysin system. Applied and Environmental Microbiology, 66(11), 5024-5029.

Gujrati, V., Kim, S., Kim, S. H., Min, J. J., Choy, H. E., Kim, S. C., Jon, S. (2014). Bioengineered bacterial outer membrane vesicles as cell-specific drug-delivery vehicles for cancer therapy. ACS Nano, 8(2), 1525-1537.

Khosa, S., Scholz, R., Schwarz, C., Trilling, M., Hengel, H., Jaeger, K. E., Smits, S. H. J., Schmitt, L. (2018). An A/U-Rich Enhancer Region Is Required for High-Level Protein Secretion through the HlyA Type I Secretion System. Applied and Environmental Microbiology, 84(1), e01163.

Kitagawa, M., Ara, T., Arifuzzaman, M., Ioka-Nakamichi, T., Inamoto, E., Toyonaga, H., Mori, H. (2005). Complete set of ORF clones of Escherichia coli ASKA library (a complete set of E. coli $\mathrm{K}-12 \mathrm{ORF}$ archive): Unique resources for biological research. DNA Research, 12(5), 291-299.

Klimentová, J., Stulík, J. (2015). Methods of isolation and purification of outer membrane vesicles from gram-negative bacteria. Microbiological Research, 170, $1-9$.

Koma, D., Yamanaka, H., Moriyoshi, K., Ohmoto, T., Sakai, K. (2012). A convenient method for multiple insertions of desired genes into target loci on the Escherichia coli chromosome. Applied Microbiology and Biotechnology, 93(2), 815-829.

Kulp, A. J., Sun, B., Ai, T., Manning, A. J., Orench-Rivera, N., Schmid, A. K., Kuehn, 

M. J. (2015). Genome-wide assessment of outer membrane vesicle production in Escherichia coli. PLoS One, 10(9), e0139200.

Lipinska, B., Zylicz, M., Georgopoulos, C. (1990). The HtrA (DegP) protein, essential for Escherichia coli survival at high temperatures, is an endopeptidase. Journal of Bacteriology, 172(4), 1791-1797.

Manning, A. J., Kuehn, M. J. (2011). Contribution of bacterial outer membrane vesicles to innate bacterial defense. BMC Microbiology, 11, 258.

McBroom, A. J., Johnson, A. P., Vemulapalli, S., Kuehn, M. J. (2006). Outer membrane vesicle production by Escherichia coli is independent of membrane instability. Journal of Bacteriology, 188(15), 5385-5392.

Ojima, Y., Yamaguchi, K., Taya, M. (2018). Quantitative evaluation of eecombinant protein packaged into outer membrane vesicles of Escherichia coli cells. Biotechnology Progress, 34(1), 51-57.

Roier, S., Zingl, F. G., Cakar, F., Durakovic, S., Kohl, P., Eichmann, T. O., Klug, L., Gadermaier, B., Weinzerl, K., Prassl, R., Lass, A., Daum, G., Reidl, J., Feldman, M. F., Schild, S. (2016). A novel mechanism for the biogenesis of outer membrane vesicles in Gram-negative bacteria. Nature Communications, 7, 10515.

Schwechheimer, C., Kuehn, M. J. (2013). Synthetic effect between envelope stress and lack of outer membrane vesicle production in Escherichia coli. Journal of Bacteriology, 195(18), 4161-4173.

Schwechheimer, C., Kuehn, M. J. (2015). Outer-membrane vesicles from Gram-negative bacteria: Biogenesis and functions. Nature Reviews Microbiology, $13(10), 605-619$.

Schwechheimer, C., Rodriguez, D. L., Kuehn, M. J. (2015). NlpI-mediated modulation 
of outer membrane vesicle production through peptidoglycan dynamics in Escherichia coli. Microbiologyopen, 4(3), 375-389.

Sevastsyanovich, Y. R., Leyton, D. L., Wells, T. J., Wardius, C. A., Tveen-Jensen, K., Morris, F. C., Knowles, T. J., Cunningham, A. F., Cole, J. A., Henderson, I. R. (2012). A generalised module for the selective extracellular accumulation of recombinant proteins. Microbial Cell Factories, 11, 69.

Toyofuku, M., Nomura, N., Eberl, L. (2019). Types and origins of bacterial membrane vesicles. Nature Reviews Microbiology, 17(1), 13-24.

9 Voulgaris, I., Finka, G., Uden, M., Hoare, M. (2015). Enhancing the selective

Wang, M., Huang, M., Zhang, J., Ma, Y., Li, S., Wang, J. (2017). A novel secretion and online-cleavage strategy for production of cecropin A in Escherichia coli. Scientific Reports, 7(1), 7368. 
2 Fig. 1 Cell growth and relative outer membrane vesicle (OMV) production in each

3 Escherichia coli strain. (A) $\mathrm{OD}_{660}$ of each E. coli strain after 24 h of culture. (B) SDS-PAGE analysis of OMVs isolated from each E. coli strain. (C) Relative OMV production by each $E$. coli strain. OMV production was normalized to that by the wild-type (WT). Vertical bars indicate standard deviations (calculated from more than three independent experiments).

(a)

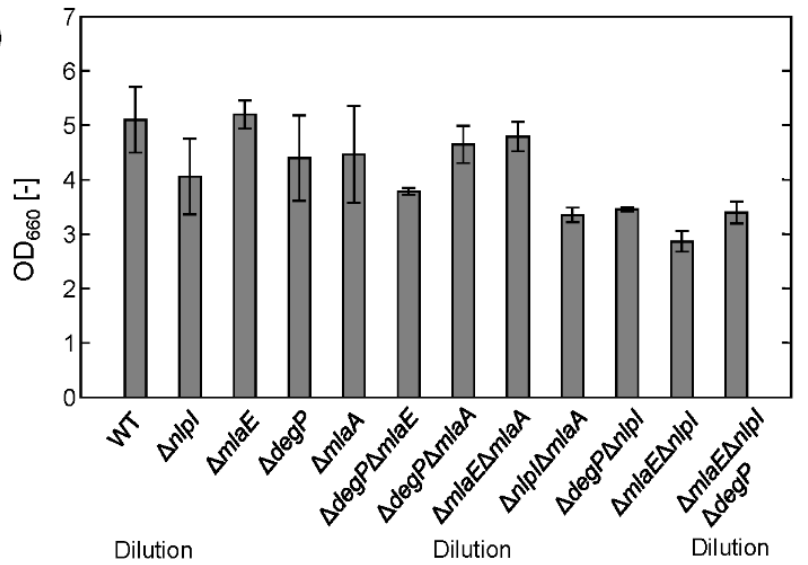

(b)

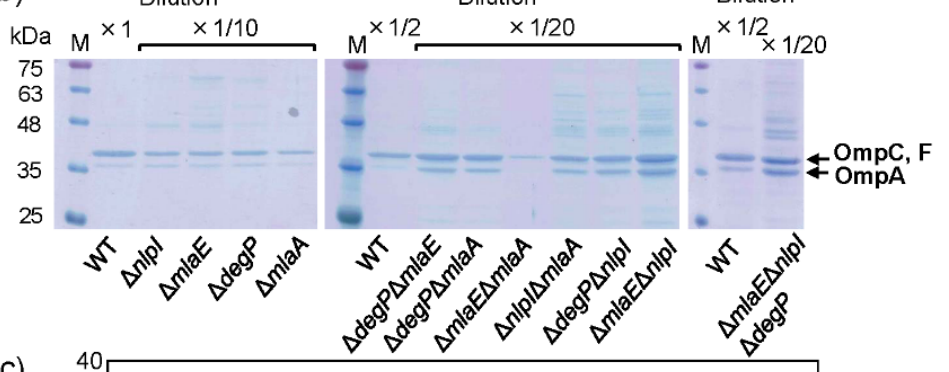

(c)

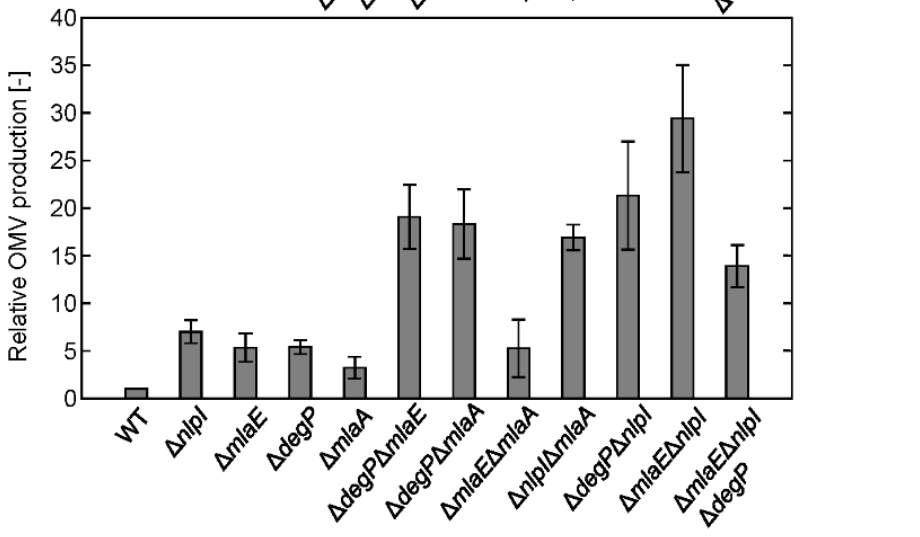


1 Fig. 2 Transmission electron microscopy images of OMVs isolated from E. coli WT and $2 \Delta m l a E \Delta n l p I$ strains. The OMVs were stained with uranyl acetate.

3
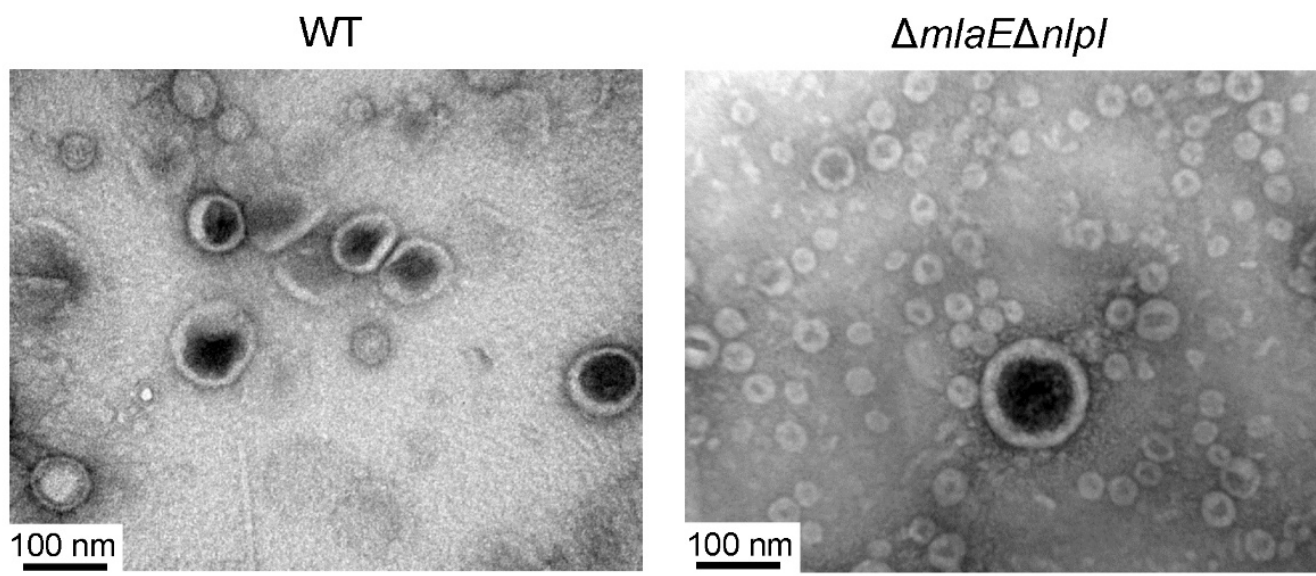
1 Fig. 3 Cell growth and relative OMV production by each E. coli strain. (A) OD 660 of each E. coli strain after $24 \mathrm{~h}$ in culture. (B) Relative OMV production by each $E$. coli strain. OMV production was normalized to that by the WT. Vertical bars indicate standard deviations (calculated from more than three independent experiments).

(A)

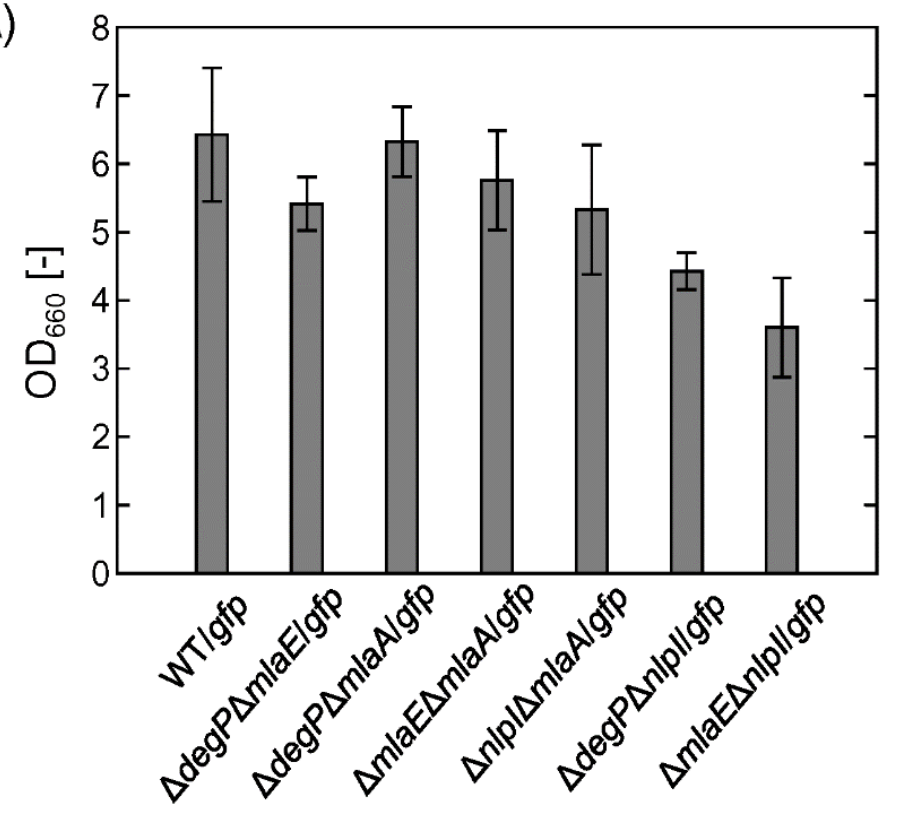

(B)

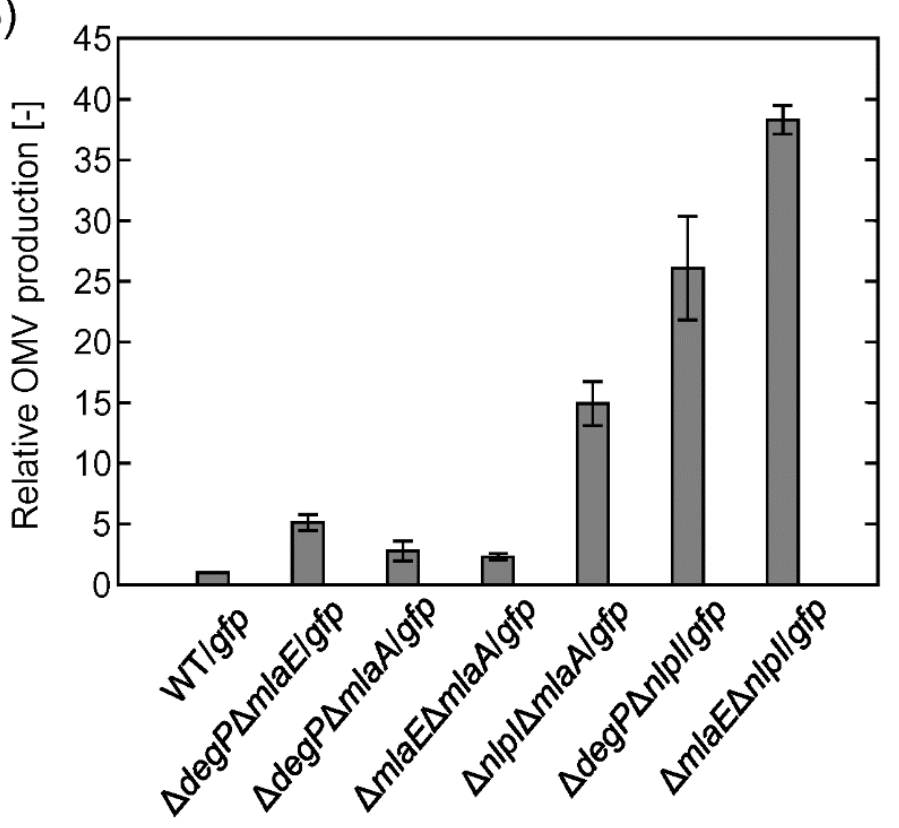


1 Fig. 4 Green fluorescent protein (GFP) secretion in the OMV fraction isolated from

each E. coli strain. (A) Western blot analysis of secreted GFP. Hybridization was performed using an anti-His-tag primary antibody. (B) Quantitative evaluation of GFP secretion in OMV fractions of respective E. coli strains. Values were quantified on the basis of a GFP standard sample. Vertical bars indicate standard deviations (calculated from more than three independent experiments).
(A)

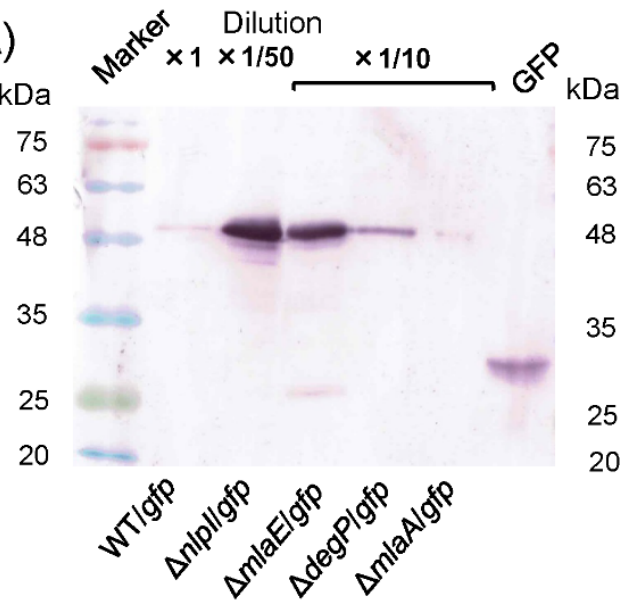

(B)

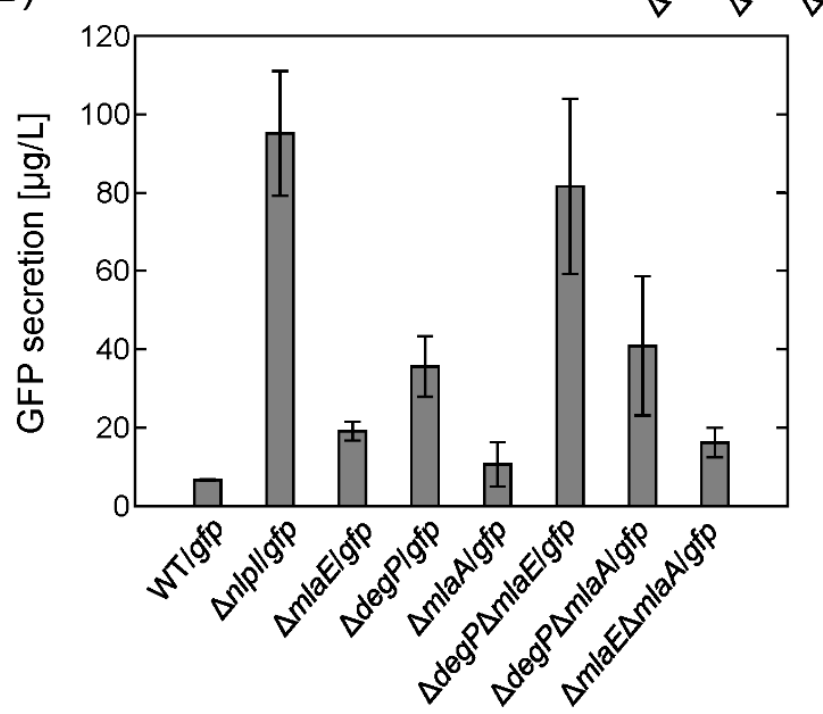

Dilution
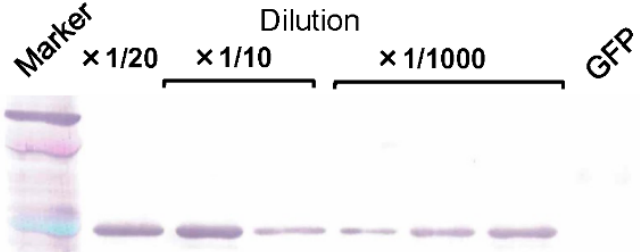

5

0
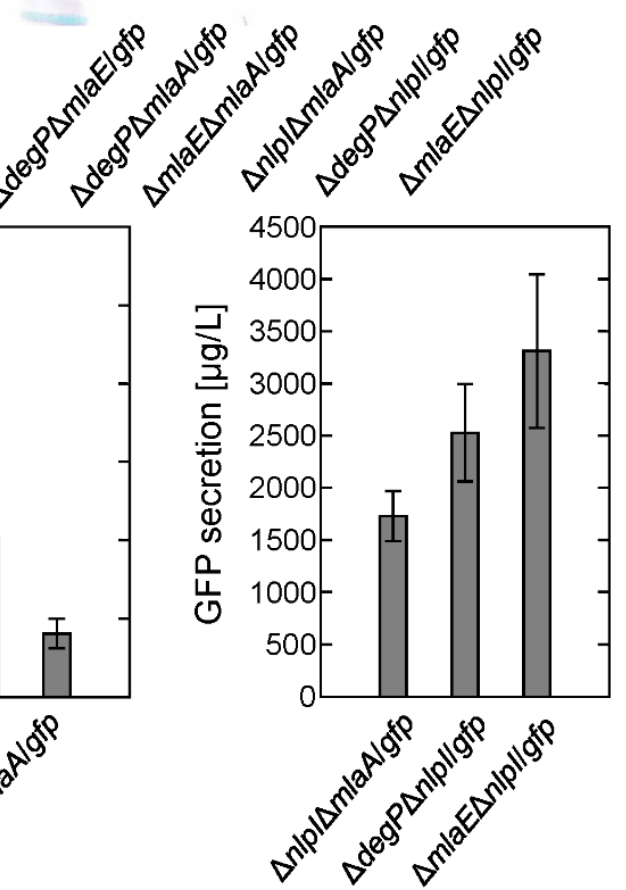
1 Supplementary Fig. S1 Western blot analysis of GFP produced in each E. coli strain.

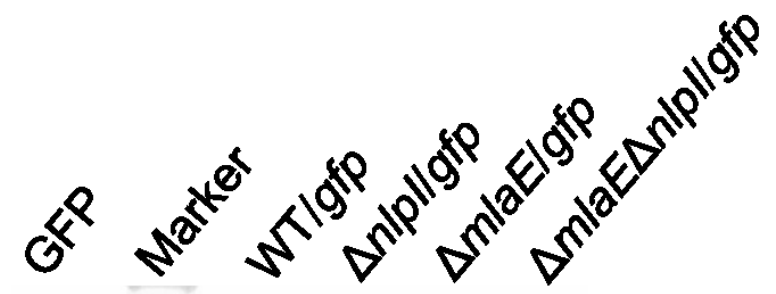


Table 1 E. coli strains and plasmid used in this study

\begin{tabular}{|c|c|c|}
\hline Strains & Note & Reference \\
\hline \multicolumn{3}{|l|}{ E. coli } \\
\hline BW25113 & rrnBT14 $\Delta l a c Z \mathrm{WJ} 16$ hsdR514 $\Delta a r a B A D A H 33$ & Baba et al., 2006 \\
\hline$\Delta n l p I(J W 3132)$ & BW25113, $\Delta n l p I:: F R T-K m-F R T$ & Baba et al., 2006 \\
\hline$\triangle \operatorname{deg} P(\mathrm{JW} 0157)$ & BW25113, $\triangle \operatorname{deg} P:: F R T-K m-F R T$ & Baba et al., 2006 \\
\hline$\Delta m l a E(\mathrm{JW} 3161)$ & BW25113, $\Delta m l a E:: F R T-K m-F R T$ & Baba et al., 2006 \\
\hline$\Delta m l a A(\mathrm{JW} 2343)$ & BW25113, $\triangle m l a A:: F R T-K m-F R T$ & Baba et al., 2006 \\
\hline$\Delta d e g P \Delta m l a E$ & $\triangle d e g P \Delta m l a E:: F R T-K m-F R T$ & This study \\
\hline$\Delta \operatorname{deg} P \Delta m l a A$ & $\triangle d e g P \Delta m l a A:: F R T-K m-F R T$ & This study \\
\hline$\Delta m l a E \Delta m l a A$ & $\Delta m l a E \Delta m l a A:: F R T-K m-F R T$ & This study \\
\hline$\Delta n l p I \Delta m l a A$ & $\Delta n l p I \Delta m l a A:: F R T-K m-F R T$ & This study \\
\hline$\Delta d e g P \Delta n l p I$ & $\Delta d e g P \Delta n l p I:: F R T-K m-F R T$ & This study \\
\hline$\Delta m l a E \Delta n l p I$ & $\Delta m l a E \Delta n l p I:: F R T-K m-F R T$ & This study \\
\hline \multicolumn{3}{|l|}{ Plasmid } \\
\hline ASKA-omp $W-g f p$ & $\begin{array}{l}\text { pCA24N carrying omp } W \text { and } g f p \text { under } \mathrm{P}_{\mathrm{T} 5-\mathrm{lac}} \\
\text { control, } \mathrm{Cm}^{\mathrm{r}}\end{array}$ & $\begin{array}{l}\text { Kitagawa et al. } \\
2005\end{array}$ \\
\hline
\end{tabular}


Table 2 Summary of GFP secretion and production, cell growth, relative OMV production of each E. coli strain

\begin{tabular}{lcccc}
\hline & WT/gfp & $\Delta n l p I / g f p$ & $\Delta m l a E / g f p$ & $\Delta m l a E \Delta n l p I / g f p$ \\
\hline GFP secretion in the medium $[\mu \mathrm{g} / \mathrm{L}]$ & $6.6 \pm 0.2$ & $95.1 \pm 16$ & $35.6 \pm 7.7$ & $3309 \pm 736$ \\
GFP prodution in the cells $[\mathrm{mg} / \mathrm{L}]$ & $269 \pm 37$ & $420 \pm 179$ & $400 \pm 110$ & $261 \pm 86$ \\
Pecentage of secreted protein [\%] & 0.003 & 0.023 & 0.009 & 1.268 \\
Relative OMV production (SDS-PAGE) [-] & 1 & $10.6 \pm 3.4$ & $1.7 \pm 0.3$ & $38.3 \pm 1.2$ \\
OD $_{660}[-]$ & $6.4 \pm 0.6$ & $5.0 \pm 0.3$ & $5.8 \pm 0.8$ & $3.4 \pm 0.4$ \\
\hline
\end{tabular}


Table 3 Selection of heterologous proteins secreted by $E$. coli cells

\begin{tabular}{|c|c|c|c|c|}
\hline Secretion system & Mechanism & Secretion product & Extracellular titer & References \\
\hline Type I & HlyA & $\mathrm{scFv}$ & $2 \mathrm{mg} / \mathrm{L}$ & Fernández et al. 2000 \\
\hline Type I & HlyA & Interferon alpha 2 & $6 \mathrm{mg} / \mathrm{L}$ & Khosa et al. 2018 \\
\hline Type I & TliDEF, HlyA & Lipase & $3 \mathrm{mg} / \mathrm{L}$ & Khosa et al. 2018 \\
\hline $\begin{array}{l}\text { Type II and } \\
\text { generalized two-step }\end{array}$ & TatExpress & Human growth hormone & $30 \mathrm{mg} / \mathrm{L}$ & Browning et al. 2017 \\
\hline $\begin{array}{l}\text { Type II and } \\
\text { generalized two-step }\end{array}$ & Leaky OM & $\begin{array}{l}\text { Human parathyroid hormone } \\
\text { (9.4 kDa with thioredoxin) }\end{array}$ & $680 \mathrm{mg} / \mathrm{L}$ & Chen et al. 2014 \\
\hline $\begin{array}{l}\text { Type II and } \\
\text { generalized two-step }\end{array}$ & Leaky OM & $\mathrm{Fab}$ & $6 \mathrm{~g} / \mathrm{L}$ & Voulgaris et al. 2015 \\
\hline Type V & Type V & Pertactin & $1 \mathrm{mg} / \mathrm{L}$ & Sevastsyanovich et al. 2012 \\
\hline Type VIII & Type VIII & Cecropin A & $\begin{array}{l}294 \mathrm{mg} / \mathrm{L} \\
\text { (after purification) }\end{array}$ & Wang et al. 2017 \\
\hline OMVs & Leaky OM & GFP & $3.3 \mathrm{mg} / \mathrm{L}$ & This study \\
\hline
\end{tabular}

